\title{
INEXISTENCIA GLOBAL PARA UNA ECUACIÓN DEL CALOR DEGENERADA NO LINEAL \\ Teófanes Quispe Méndez*
}

Resumen: En el presente trabajo, estudiamos la singularidad en tiempo finito de las soluciones del problema mixto para un tipo de ecuación del calor degenerada no lineal con una función de Lewis generalizada.

Palabras clave: Singularidad de soluciones, Inexistencia global, Ecuación del calor degenerada no lineal, Ecuación de evolución.

\section{NONEXISTENCE OF GLOBAL SOLUTIONS FOR A NONLINEAR DEGENERATE HEAT EQUATION}

\begin{abstract}
In present work, we study the blow-up finite time of solutions to the mixed problem for a type of nonlinear degenerate heat equation with a generalized Lewis function.

Key words: Blow-up of solutions, Nonexistence global, Nonlinear degenerate heat equation, Evolution equation.
\end{abstract}

\section{Introducción}

En este artículo consideramos el siguiente problema de valores inicial y frontera con una función de Lewis generalizada $\alpha(x, t)$ :

$$
\begin{cases}\alpha(x, t) u_{t}-\beta(t) \Delta u_{t}-\Delta_{p} u=f(u) & , x \in \Omega, t \geq 0 \\ u=0 & , x \in \partial \Omega, t \geq 0 \\ u(x, 0)=u_{0}(x) & , x \in \Omega\end{cases}
$$

donde $\Omega$ es un conjunto abierto y acotado de $\mathbb{R}^{n}$ con frontera bien regular $\partial \Omega, \Delta$ es el operador laplaciano, $\alpha(x, t)$ es una función real positiva para $x \in \Omega, t \geq 0, \beta(t)$ es una función real positiva para $t \geq 0, f(s)$ es una función real no lineal para $s \in \mathbb{R}$, y el operador $p$-laplaciano $-\Delta_{p}$ definido para $p \geq 2$ :

$$
-\Delta_{p} u:=-\sum_{i=1}^{n} \frac{\partial}{\partial x_{i}}\left(\left|\frac{\partial u}{\partial x_{i}}\right|^{p-2} \frac{\partial u}{\partial x_{i}}\right) .
$$

Las ecuaciones de tipo (1.1) son utilizadas para modelar diversos fenómenos y procesos en mecánica, física, tecnología, biología y muchas otras áreas. Por ejemplo, describe el proceso de conducción en plasma, filtración de gases y líquidos en medios porosos, reacciones químicas, procesos de crecimiento y migración de poblaciones, y entre otros [7].

En el caso $p=2$. la ecuación en (1.1) se reduce a la ecuación clásica del calor, y se tiene sobre ello una literatura bastante impresionante en cuanto al estudio de existencia y no existencia de las soluciones globales, y las propiedades de estas, citamos algumos de ellos $[1,2,3,4,8,10]$. El caso

\footnotetext{
${ }^{*}$ Profesor de la Facultad de Ciencias Matemáticas de la Universidad Nacional Mayor de San Marcos. e-mail: tquispem 0 mminsm.edu.pe
} 
$p>2$, no se tiene muchos resultados conocidos. Tsutsumi [9], obtiene existencia y no existencia de las soluciones globales con los métodos de aproximación de Galerkin y del pozo potencial, cuando $\alpha \equiv 1, \beta \equiv 0$ y $f(u):= \pm u^{\sigma-1}, \sigma \geq 2$. Messaoudi [5], obtiene singularidad en tiempo finito con energía inicial no positiva con el método de concavidad, cuando $\alpha \equiv 1$ y $\beta \equiv 0$. Zhou [11], obtiene singularidad en tiempo finito con energía inicial positiva restringida y con energía inicial no positiva con el método de concavidad, cuando $\beta \equiv 0$. Quispe Méndez [6], obtiene existencia de las soluciones locales con el método de aproximación de Galerkin y las estimativas de Tartar.

En este trabajo probaremos la propiedad de singularidad en tiempo finito de las soluciones del problema (1.1) con energía inicial no positiva y con energía inicial positiva restringida, por el método de concavidad [4]. También se obtiene las estimativas para el tiempo de vida de las soluciones.

\section{Preliminares}

En esta sección daremos algunas notaciones, conceptos, lemas y teoremas sin demostración que serán utilizadas en el desarrollo del presente trabajo.

Sea $\Omega$ un conjunto abierto y acotado de $\mathbb{R}^{n}$ con frontera bien regular $\partial \Omega$. Denotamos el producto interno y la norma de $L^{2}(\Omega)$ y $L^{p}(\Omega)$, con $(.,$.$) y |.|_{p}$, respectivamente, para $1 \leq p \leq \infty$. Además $((.,)$.$) y \|$.$\| , denotarán el producto interno y la norma de H_{0}^{1}(\Omega)$, donde $((u, v)):=\int_{\Omega} \nabla u(x) \cdot \nabla v(x) d x$ es la forma de Dirichlet. En el espacio de Sobolev $W_{0}^{1, p}(\Omega)$ usamos la norma

$$
\|u\|_{1, p}:=\left(\sum_{i=1}^{n}\left|\frac{\partial u}{\partial x_{i}}\right|_{p}^{p}\right)^{\frac{1}{p}} .
$$

Sea $X$ un espacio de Banach, $0<T \leq \infty$ y $1 \leq p \leq \infty$. Representamos con $L^{p}(0, T ; X)$ al espacio de Banach de las funciones vectoriales $u:] 0, T[\longrightarrow X$ tales que son medibles $\mathrm{y}$ $\|u(t)\|_{X} \in L^{p}(0, T)$, con la norma

$$
\begin{gathered}
\|u\|_{L^{p}(0, T ; X)}:=\left(\int_{0}^{T}\|u(t)\|_{X}^{p} d t\right)^{\frac{1}{p}}, 1 \leq p<\infty, \\
\|u\|_{L^{\infty}(0, T ; X)}:=\sup _{0<t<T} \operatorname{ess}\|u(t)\|_{X} ; p=\infty .
\end{gathered}
$$

Denotamos $v^{\prime}:=\frac{\partial v}{\partial t}=v_{t}$ y $v(t)(x):=v(x, t)$.

Hipótesis. Imponemos sobre las funciones reales $\alpha(x, t), \beta(t)$ y $f(s)$ las siguientes condiciones:

(H1) $\alpha \in W^{1, \infty}\left(0, \infty ; L^{\infty}(\Omega)\right)$ y $\alpha(x, t) \geq \alpha_{0}>0, \forall(x, t) \in \Omega \times\left[0, \infty\left[, \alpha_{0}\right.\right.$ es una constante.

$(H 2) \beta \in W^{1, \infty}(0, \infty)$ y $\beta(t) \geq \beta_{0}>0, \forall t \geq 0, \beta_{0}$ es una constante.

$(H 3) f \in C^{0}(\mathbb{R})$ y existe una constante positiva $C_{0}$ tal que

$$
|f(s)| \leq C_{0}|s|^{\sigma-1}, \forall s \in \mathbb{R},
$$

donde $2 \leq p<\sigma<\frac{p(n+2)}{n}$.

(H4) $f(s) s \geq \sigma F(s), \forall s \in \mathbb{R}$, donde $\sigma$ es la mismia constante de $(H 3)$ y $F(s):=\int_{0}^{s} f(\xi) d \xi$.

$(H 5) \quad a_{t}(x, t) \leq 0$ c.t.p. para $t \geq 0, \forall x \in \Omega$ y $\quad \beta^{\prime}(t) \leq 0$ c.t.p. para $t \geq 0$. 
(H6) $\alpha_{t}(x, t) \geq 0$ c.t.p. para $t \geq 0, \forall x \in \Omega$ y $\beta^{\prime}(t) \geq 0$ c.t.p. para $t \geq 0$.

Lema 2.1 (Desigualdad de Sobolev-Poincaré [3]). Para cada función $u \in W_{0}^{1, p}(\Omega)$, se tiene

$$
|u|_{r} \leq B_{0}\|u\|_{1, p},
$$

donde $1 \leq r \leq \frac{p m}{n-p}$ si $1 \leq p<n$ y $1 \leq r<\infty$ si $1 \leq n \leq p$. La constante $B_{0}$ depende solamente de $\Omega, n, p$ y $r$.

Lema 2.2. El operador p-laplaciano $-\Delta_{p}$ es acotado, estrictamente monótono, semicontinuo y coercivo de $W_{0}^{1, p}(\Omega)$ en $W^{-1, q}(\Omega)$,

$$
\left\langle-\Delta_{p} u, v\right\rangle=\int_{\Omega}|\nabla u|^{p-2} \nabla u \cdot \nabla v d x, \forall u, v \in W_{0}^{1, p}(\Omega)
$$

$y$

$$
\left|\left\langle-\Delta_{p} u, v\right\rangle\right| \leq\|u\|_{1, p}^{p-1}\|v\|_{1, p}, \quad \forall u, v \in W_{0}^{1, p}(\Omega),
$$

donde $W^{-1, q}(\Omega)$ es el espacio dual de $W_{0}^{1, p}(\Omega)$ y $p^{-1}+q^{-1}=1$.

Definición 2.3. Una función $u: \Omega \times[0, T] \rightarrow \mathbb{R}$ es llamada solución del problema (1.1) sobre $[0, T]$ si satisface las condiciones $(1.1)_{2}-(1.1)_{3} \mathrm{y}$ la igualdad

$$
\alpha(x, t) u_{t}-\beta(t) \Delta u_{t}-\Delta_{p} u=f(u) \text { en } L^{2}\left(0, T ; W^{-1, q}(\Omega)\right),
$$

donde $p^{-1}+q^{-1}=1$.

Teorema 2.4 (Existencia Local [6]). Supongamos que las funciones $\alpha, \beta$ y $f$ satisfacen las hipótesis $(H 1)-(H 3)$, respectivamente, y que $u_{0} \in W_{0}^{1, p}(\Omega)$. Entonces existe un único intervalo $\left[0, T_{\text {máx }}\left[\right.\right.$ con $0<T_{\text {máx }} \leq \infty$ y el problema (1.1) admite una solución u sobre $\left[0, T_{\text {máx }}[\right.$ tal que

$$
u \in L^{\infty}\left(0, T_{\text {máx }} ; W_{0}^{1, p}(\Omega)\right) \text { y } u^{\prime} \in L^{2}\left(0, T_{\text {máx }} ; H_{0}^{1}(\Omega)\right) .
$$

Lema $2.5([2])$. Supongamos que $\varphi(t)$ es una función no negativa, dos veces diferenciable y satisface la inecuación diferencial para $t \geq 0$ :

$$
\varphi^{\prime \prime}(t) \varphi(t)-(1+\mu)\left(\varphi^{\prime}(t)\right)^{2} \geq 0,
$$

donde $\mu$ es una constante positiva. Si $\varphi(0)>0 y \varphi^{\prime}(0)>0$, entonces existe un número real positivo $T_{*}$ tal que $\lim _{t \rightarrow T_{*}^{-}} \varphi(t)=+\infty$ y una cota superior de $T_{*}$ puede ser estimado por

$$
T_{*} \leq \frac{\varphi(0)}{\mu \varphi^{\prime}(\theta)}
$$

\section{EL RESULTADO PRINCIPAL}

El objetivo central del presente trabajo es discutir la propiedad de singularidad en tiempo finito de las soluciones del problema (1.1) sobre un intervalo maximal $\left[0, T_{\text {máx }}[\right.$. Para este propósito, utilizamos las técnicas desarrolladas por Levine [4] y Zhou. [11], llamada método de concavidad. 
Definición 3.1. Una solución $u$ del problema (1.1) sobre un intervalo maximal $\left[0, T_{\text {máx }}[\right.$ tiene la propiedad de explosión o singularidad en tiempo finito si

$$
T_{\operatorname{máx}}<+\infty,
$$

es decir que $u$ no puede ser extendida sobre $[0,+\infty[$ como solución del problema (1.1).

Definición 3.2. La función energía $E(t)$ del problema (1.1), se define por

$$
E(t):=\frac{1}{p}\|u(t)\|_{1, p}^{p}-\int_{\Omega} F(u(x, t)) d x, \quad t \geq 0 .
$$

donde

$$
F(s):=\int_{0}^{s} f(\xi) d \xi
$$

Lema 3.3. Supongamos que se cumplen las hipótesis $(H 1)-(H 3)$. Si u es una solución del problema (1.1) sobre $\left[0, T_{\text {máx }}\left[\right.\right.$ con dato inicial $u_{0} \in W_{0}^{1, p}(\Omega)$, entonces

$$
E(t)+\int_{0}^{t}\left|\sqrt{\alpha(s)} u^{\prime}(s)\right|_{2}^{2} d s+\int_{0}^{t} \beta(s) \| u^{\prime}(s) H^{2} d s=E(0)
$$

donde $E(0)$ es la energía inicial definida por

$$
E(0):=\frac{1}{p}\left\|u_{0}\right\|_{1, p}^{p}-\int_{\Omega} F\left(u_{0}(x)\right) d x .
$$

Demostración. Multiplicando a la ecuación $(1.1)_{1}$ por $u_{t}$, integrando sobre $\Omega$, aplicando el Lema 2.2 y el Teorema de la Divergencia, obtenemos

$$
E^{\prime}(t)+\left|\sqrt{\alpha(t)} u^{\prime}(t)\right|_{2}^{2}+\beta(t)\left\|u^{\prime}(t)\right\|^{2}=0 .
$$

De aquí, se obtiene el resultado (3.1).

Lema 3.4. Sea la función definida para $\lambda \geq 0$ :

$$
g(\lambda):=\frac{1}{p B_{0}^{p}} \lambda^{p}-\frac{C_{0}}{\sigma} \lambda^{\sigma},
$$

donde $B_{0}$ es la constante óptima de la desigualdad de Sobolev-Poincaré; $C_{0}, p$ y $\sigma$ son constantes de la hipótesis (H3). Entonces

(i) g es estrictamente creciente en $\left[0, \lambda_{0}[\right.$,

(ii) g toma su valor máximo $E_{0}$ en $\lambda_{0}$,

(iii) $g$ es estrictamente decreciente en $] \lambda_{0}, \infty[$,

donde

$$
\lambda_{0}:=\left(C_{0} B_{0}^{p}\right)^{\frac{-1}{\sigma-p}} \text { y } \quad E_{0}:=\left(\frac{1}{p}-\frac{1}{\sigma}\right)\left(C_{0} B_{0}^{\sigma}\right)^{\frac{-p}{\sigma-p}} .
$$

Demostración. Es inmediato.

Lema 3.5. Supongamos que se cumplen las hipótesis $(H 1)-(H 3)$. Si u es una solución del problema (1.1) sobre $\left[0, T_{\text {máx }}\left[\right.\right.$ con dato inicial $u_{0} \in W_{0}^{1, p}(\Omega)$. y que satisface

$$
\left|u_{0}\right|_{\sigma}>\lambda_{0} \text { y } E(0)<E_{0} .
$$


entonces

$$
|u(t)|_{\sigma}>\lambda_{0} \quad y \quad\|u(t)\|_{1, p}>\left(C_{0} B_{0}^{\sigma}\right)^{\frac{-1}{\sigma-p}}, \quad t \geq 0 .
$$

Demostración. Por (3.2). $E(t)$ es una función no creciente y $E(t) \leq E(0), t \geq 0$. Por la Desigualdad de Sobolev-Poincaré y $(H 3)$, resulta

$$
\begin{aligned}
E(t) & \geq \frac{1}{p B_{0}^{p}}|u(t)|_{\sigma}^{p}-\frac{C_{0}}{\sigma}|u(t)|_{\sigma}^{\sigma} \\
& =g\left(|u(t)|_{\sigma}\right), \quad t \geq 0 .
\end{aligned}
$$

Así se tiene

$$
g\left(|u(t)|_{\sigma}\right) \leq E(t) \leq E(0)<g\left(\lambda_{0}\right):=E_{0}, \quad t \geq 0 .
$$

Por (3.6), existe $\lambda_{1}>\lambda_{0}$ tal que $g\left(\lambda_{1}\right)=E(0)$. A continuación probemos que

$$
|u(t)|_{\sigma} \geq \lambda_{1}, t \geq 0
$$

Por el absurdo. Supongamos que existe $t_{0}>0$ tal que $\lambda_{0}<\left|u\left(t_{0}\right)\right|_{\sigma}<\lambda_{1}$. Desde que $g$ es estrictamente decreciente en $] \lambda_{0}, \infty[$, resulta

$$
E(0)=g\left(\lambda_{1}\right)<g\left(\left|u\left(t_{0}\right)\right|_{\sigma}\right)<g\left(\lambda_{0}\right)
$$

y esto es una contradicción con (3.6). Por tanto se cumple (3.7) y $|u(t)|_{\sigma}>\lambda_{0}, t \geq 0$. Por la Desigualdad de Sobolev-Poincaré, resulta

$$
\|u(t)\|_{1, p} \geq B_{0}^{-1}|u(t)|_{\sigma}>B_{0}^{-1} \lambda_{0}=\left(C_{0} B_{0}^{\sigma}\right)^{\frac{-1}{\sigma-p}} .
$$

Por tanto, se obtiene (3.5).

Teorema 3.6 (Inexistencia Global). Supongamos que se cumplen las hipótesis $(H 1)-(H 4)$, y (H5) ó (H6). Si u es una solución del problema (1.1) sobre el intervalo maximal [0, $T_{\operatorname{máx}}$ [ con dato inicial $u_{0} \in W_{0}^{1, p}(\Omega)$, y que satisface

$$
\left|u_{0}\right|_{\sigma}>\lambda_{0} \quad y \quad E(0)<E_{0},
$$

entonces $T_{\operatorname{máx}}<+\infty$, es decir que u no puede ser extendida sobre $[0,+\infty[$ como solución del problema (1.1). Además el tiempo finito $T_{\operatorname{máx}}$ es estimado por

$$
T_{\operatorname{máx}} \leq \frac{8\left(\left|\sqrt{\alpha(0)} u_{0}\right|_{2}^{2}+\beta(0)\left\|u_{0}\right\|^{2}\right)}{(\sigma-2)^{2}\left(E_{0}-E(0)\right)}
$$

Demostración. Consideremos dos casos.

Caso 1: Supongamos que se cumple (H5). Para este caso definamos la función explosión para $t \in\left[0, T_{0}\right]:$

$$
\begin{aligned}
\varphi(t):= & \int_{0}^{t}|\sqrt{\alpha(s)} u(s)|_{2}^{2} d s+\int_{0}^{t}(s-t)\left(\alpha^{\prime}(s) u(s), u(s)\right) d s \\
& +\int_{0}^{t} \beta(s)\|u(s)\|^{2} d s+\int_{0}^{t}(s-t) \beta^{\prime}(s)((u(s), u(s))) d s \\
& +\left(T_{0}-t\right)\left[\left|\sqrt{\alpha(0)} u_{0}\right|_{2}^{2}+\beta(0)\left\|u_{0}\right\|^{2}\right] \\
& +\delta \cdot\left(t+\tau_{0}\right)^{2}
\end{aligned}
$$


donde $\tau_{0}, T_{0}$ y $\delta$ son constantes positivas que serán determinadas más tarde.

Por diferenciación de (3.9), resulta

$$
\begin{aligned}
\varphi^{\prime}(t)=\mid & \left.\sqrt{\alpha(t)} u(t)\right|_{2} ^{2}-\int_{0}^{t}\left(\alpha^{\prime}(s) u(s), u(s)\right) d s \\
& +\beta(s)\|u(t)\|^{2}-\int_{0}^{t} \beta^{\prime}(s)((u(s), u(s))) d s \\
& -\left[\left|\sqrt{\alpha(0)} u_{0}\right|_{2}^{2}+\beta(0)\left\|u_{0}\right\|^{2}\right]+2 \delta\left(t+\tau_{0}\right) .
\end{aligned}
$$

Notemos que se cumplen

$$
\begin{aligned}
2 \int_{0}^{t}\left(\alpha(s) u^{\prime}(s), u(s)\right) d s= & |\sqrt{\alpha(t)} u(t)|_{2}^{2}-\left|\sqrt{\alpha(0)} u_{0}\right|_{2}^{2} \\
& -\int_{0}^{t}\left(\alpha^{\prime}(s) u(s), u(s)\right) d s
\end{aligned}
$$

y

$$
\begin{aligned}
2 \int_{0}^{t} \beta(s)\left(\left(u^{\prime}(s), u(s)\right)\right) d s= & \beta(t)\|u(t)\|^{2} d s-\beta(0)\left\|u_{0}\right\|^{2} \\
& -\int_{0}^{t} \beta^{\prime}(s)((u(s), u(s))) d s .
\end{aligned}
$$

Por (3.11) y (3.12), se tiene de (3.10)

$$
\begin{aligned}
\varphi^{\prime}(t)= & 2 \int^{t}\left(\alpha(s) u^{\prime}(s), u(s)\right) d s+2 \int_{0}^{t} \beta(s)\left(\left(u^{\prime}(s), u(s)\right)\right) d s \\
& +2 \delta\left(\tau_{0}\right) .
\end{aligned}
$$

Diferenciando (3.13), utilizando la ecuación $(1.1)_{1}$, Lema 2.2 y el Teorema de la Divergencia, se obtiene

$$
\varphi^{\prime \prime}(t)=2(f(u(t)), u(t))-2\|u(t)\|_{1, p}^{p}+2 \delta .
$$

Por $(H 4),(3.1)$ y $(3.5)$, resulta

$$
\begin{aligned}
\varphi^{\prime \prime}(t) \geq & 2 \sigma \int_{\Omega} F(u(x, t)) d x-2\|u(t)\|_{1, p}^{p}+2 \delta \\
= & 2\left(\frac{\sigma}{p}-1\right)\|u(t)\|_{1, p}^{p}-2 \sigma E(0)+2 \delta \\
& +2 \sigma \int_{0}^{t}\left|\sqrt{\alpha(s)} u^{\prime}(s)\right|_{2}^{2} d s+2 \sigma \int_{0}^{t} \beta(s)\left\|u^{\prime}(s)\right\|^{2} d s \\
> & 2 \sigma\left(\frac{1}{p}-\frac{1}{\sigma}\right)\left(C_{0} B_{0}^{\sigma}\right)^{\frac{-p}{\sigma-p}}-2 \sigma E(0)+2 \delta \\
& +2 \sigma \int_{0}^{t}\left|\sqrt{\alpha(s)} u^{\prime}(s)\right|_{2}^{2} d s+2 \sigma \int_{0}^{t} \beta(s)\left\|u^{\prime}(s)\right\|^{2} d s \\
= & 2 \sigma\left(E_{0}-E(0)\right)+2 \delta \\
& +2 \sigma \int_{0}^{t}\left|\sqrt{\alpha(s)} u^{\prime}(s)\right|_{2}^{2} d s+2 \sigma \int_{0}^{t} \beta(s)\left\|u^{\prime}(s)\right\|^{2} d s
\end{aligned}
$$

Tomando $\delta=2\left(E_{0}-E(0)\right)$ y desde que $\sigma>2$. se tiene

$$
\varphi^{\prime \prime}(t)>(\sigma+2) \delta+(\sigma+2)\left[\int_{0}^{t}\left|\sqrt{a(s)} u^{\prime}(s)\right|_{2}^{2} d s+\int_{0}^{t} \beta(s)\left\|u^{\prime}(s)\right\|^{2} d s\right] .
$$


De (3.9), (3.13) y (3.15), se obtienen

$$
\begin{aligned}
& \varphi(0)=T_{0}\left[\left|\sqrt{\alpha(0)} u_{0}\right|_{2}^{2}+\beta(0)\left\|u_{0}\right\|^{2}\right]+\delta \tau_{0}^{2}>0 \\
& \varphi^{\prime}(0)=2 \delta \tau_{0}>0 \\
& \varphi^{\prime \prime}(t)>(\sigma+2) \delta>0, \forall t \geq 0
\end{aligned}
$$

Por (3.16), resulta que $\varphi(t)$ y $\varphi^{\prime}(t)$ son funciones positivas. Desde que $\alpha_{t}(x, t) \leq 0$ c.t.p. para $t \geq 0, \forall x \in \Omega$ y $\beta^{\prime}(t) \leq 0$ c.t.p. para $t \geq 0$, de (3.9), se tiene

$$
\varphi(t) \geq \int_{0}^{t}|\sqrt{\alpha(s)} u(s)|_{2}^{2} d s+\int_{0}^{t} \beta(s)\|u(s)\|^{2} d s+\delta\left(t+\tau_{0}\right)^{2} .
$$

De (3.13) y (3.15), resultan

$$
\varphi^{\prime}(t) \geq 2\left[\int_{0}^{t}\left(\alpha(s) u^{\prime}(s), u(s)\right) d s+\int_{0}^{t} \beta(s)\left(\left(u^{\prime}(s), u(s)\right)\right) d s+\delta\left(t+\tau_{0}\right)\right]
$$

y

$$
\varphi^{\prime \prime}(t) \geq(\sigma+2)\left[\int_{0}^{t}\left|\sqrt{\alpha(s)} u^{\prime}(s)\right|_{2}^{2} d s+\int_{0}^{t} \beta(s)\left\|u^{\prime}(s)\right\|^{2} d s+\delta\right] .
$$

De (3.17), (3.18) y (3.19), se obtiene $\forall(\xi, \eta) \in \mathbb{R}^{2}$

$$
\begin{aligned}
\varphi(t) \xi^{2}+\varphi^{\prime}(t) \xi \eta+\frac{\varphi^{\prime \prime}(t)}{\sigma+2} \eta^{2}= & \int_{0}^{t}\left|\sqrt{\alpha(s)} u(s) \xi+\sqrt{\alpha(s)} u^{\prime}(s) \eta\right|_{2}^{2} d s \\
& +\int_{0}^{t} \beta(s)\left\|u(s) \xi+u^{\prime}(s) \eta\right\|^{2} d s \\
& +\delta\left[\left(t+\tau_{0}\right) \xi+\eta\right]^{2} \\
\geq & 0 .
\end{aligned}
$$

Por propiedad de las formas cuadráticas, de (3.20) resulta

$$
\varphi^{\prime \prime}(t) \varphi(t)-(1+\mu)\left(\varphi^{\prime}(t)\right)^{2} \geq 0
$$

donde $\mu:=\frac{\sigma-2}{4}$.

Por (3.16) y (3.21), la función $\varphi$ satisface las condiciones del Lema 2.5. Entonces existe un tiempo finito $T_{*}$ tal que $\lim _{t \rightarrow T_{*}^{-}} \varphi(t)=+\infty$ y la cota superior de $T_{*}$ es estimada por

$$
T_{*} \leq T_{0}\left(\tau_{0}\right),
$$

donde

$$
T_{0}\left(\tau_{0}\right)=\frac{2 T_{0}\left(\tau_{0}\right)\left[\left|\sqrt{\alpha(0)} u_{0}\right|_{2}^{2}+\beta(0)\left\|u_{0}\right\|^{2}\right]+2 \delta \tau_{0}^{2}}{(\sigma-2) \delta \tau_{0}}
$$

y esto es equivalente a la relación

$$
T_{0}\left(\tau_{0}\right)=\frac{2 \delta \tau_{0}^{2}}{(\sigma-2) \delta \tau_{0}-2\left[\left|\sqrt{\alpha(0)} u_{0}\right|_{2}^{2}+\beta(0)\left\|u_{0}\right\|^{2}\right]} .
$$

El valor de $T_{0}$ en (3.22) debe ser el óptimo, para ello debemos escoger el valor adecuado de $\tau_{0}$ en el intervalo

$$
] \tau_{1},+\infty[\text {, }
$$




\section{INEXISTENCIA GLOBAL PARA UNA ECUACIÓN DEL CALOR DEGENERADA NO LINEAI}

donde

$$
\tau_{1}:=\frac{\left|\sqrt{\alpha(0)} u_{0}\right|_{2}^{2}+\beta(0)\left\|u_{0}\right\|^{2}}{(\sigma-2)\left(E_{0}-E(0)\right)},
$$

y esto se logra minimizando en el intervalo (3.23) la función

$$
T(\tau):=\frac{2 \delta \tau^{2}}{(\sigma-2) \delta \tau-2\left[\left|\sqrt{\alpha(0)} u_{0}\right|_{2}^{2}+\beta(0)\left\|u_{0}\right\|^{2}\right]},
$$

donde $\delta=2\left(E_{0}-E(0)\right)$. De $(3.24)$, se obtienen

$$
\tau_{0}=\frac{4\left[\left|\sqrt{\alpha(0)} u_{0}\right|_{2}^{2}+\beta(0)\left\|u_{0}\right\|^{2}\right]}{(\sigma-2) \delta}
$$

y

$$
T_{0}\left(\tau_{0}\right)=T_{\min }:=T\left(\tau_{0}\right)=\frac{8\left(\left|\sqrt{\alpha(0)} u_{0}\right|_{2}^{2}+\beta(0)\left\|u_{0}\right\|^{2}\right)}{(\sigma-2)^{2}\left(E_{0}-E(0)\right)} .
$$

Así se tiene la estimativa (3.8). Por último, desde que [0, $T_{\text {máx }}$ [ es el intervalo maximal de las soluciones del problema (1.1), resulta que $T_{\operatorname{máx}}=T_{*}$. Con esto se finaliza la demostración del Caso 1.

Caso 2: Supongamos que se cumple $(H 6)$. Para este caso definamos la función explosión para $t \in\left[0, T_{0}\right]$ :

$$
\begin{aligned}
\varphi(t):=\int_{0}^{t}|\sqrt{\alpha(s)} u(s)|_{2}^{2} d s+\int_{0}^{t} \beta(s)\|u(s)\|^{2} d s \\
+\left(T_{0}-t\right)\left[\left|\sqrt{\alpha(0)} u_{0}\right|_{2}^{2}+\beta(0)\left\|u_{0}\right\|^{2}\right] \\
+\delta\left(t+\tau_{0}\right)^{2},
\end{aligned}
$$

donde $\tau_{0}, T_{0}$ y $\delta$ son constantes positivas que serán determinadas más tarde.

Por diferenciación de (3.25), resulta

$$
\begin{aligned}
\varphi^{\prime}(t)=\mid & \left.\sqrt{\alpha(t)} u(t)\right|_{2} ^{2}+\beta(s)\|u(t)\|^{2} \\
& -\left[\left|\sqrt{\alpha(0)} u_{0}\right|_{2}^{2}+\beta(0)\left\|u_{0}\right\|^{2}\right]+2 \delta\left(t+\tau_{0}\right) .
\end{aligned}
$$

Por (3.11) y (3.12), se obtiene de (3.26)

$$
\begin{aligned}
\varphi^{\prime}(t)= & 2 \int_{0}^{t}\left(\alpha(s) u^{\prime}(s), u(s)\right) d s+2 \int_{0}^{t} \beta(s)\left(\left(u^{\prime}(s), u(s)\right)\right) d s \\
& +\int_{0}^{t}\left|\sqrt{\alpha^{\prime}(s)} u(s)\right|_{2}^{2} d s+\int_{0}^{t} \beta^{\prime}(s)\|u(s)\|^{2} d s \\
& +2 \delta\left(t+\tau_{0}\right) .
\end{aligned}
$$

Diferenciando (3.27), utilizando la ecuación (1.1), Lema 2.2 y el Teorema de la Divergencia. se obtiene

$$
\begin{aligned}
\varphi^{\prime \prime}(t)= & 2(f(u(t)), u(t))-2\|u(t)\|_{1, p}^{p}+2 \delta \\
& +\left|\sqrt{\alpha^{\prime}(t)} u(t)\right|_{2}^{2}+\beta^{\prime}(t)\|u(t)\|^{2} .
\end{aligned}
$$

De aquí el resto es idéntico al Caso 1, conectando (3.28) con lo que sigue de (3.14). 
Con esto se concluye la demostración del Teorema 3.6.

Teorema 3.7 (Inexistencia Global). Supongamos que se cumplen las hipótesis $(H 1)-(H 4)$, (H6) y $p>2$. Si u es una solución del problema (1.1) sobre el intervalo maximal [0, $T_{\text {máx}}[$ con dato inicial no cero $u_{0} \in W_{0}^{1, p}(\Omega)$. y que satisface

$$
E(0) \leq 0,
$$

entonces $T_{\operatorname{máx}}<+\infty$, es decir que u no puede ser extendida sobre $[0,+\infty[$ como solución del problema (1.1). Además el tiempo finito $T_{\operatorname{máx}}$ es estimado por

$$
T_{\text {máx }} \leq \frac{2^{\frac{p}{2}} p D_{0}^{p}|\Omega|^{\frac{p-2}{2}} C_{\alpha \beta}^{\frac{p}{2}}}{(p-2)(\sigma-p) \operatorname{mín}\left\{D_{0}^{p}, 1\right\} K_{0}^{\frac{p-2}{2}}},
$$

donde

$$
\begin{gathered}
K_{0}:=\left|\sqrt{\alpha(0)} u_{0}\right|_{2}^{2}+\beta(0)\left\|u_{0}\right\|^{2}, \\
C_{\alpha \beta}:=\operatorname{máx}\left\{\|\alpha\|_{L^{\infty}\left(0, \infty ; L^{\infty}(\Omega)\right)},\|\beta\|_{L^{\infty}(0, \infty)}\right\},
\end{gathered}
$$

$|\Omega|$ es la medida de $\Omega$ y $D_{0}$ es la constante óptima de la desigualdad de Sobolev-Poincaré $|u|_{p} \leq$ $D_{0}\|u\|_{1, p}, \forall u \in W_{0}^{1, p}(\Omega)$.

Demostración. Definamos la función explosión para $t \geq 0$ :

$$
\varphi(t):=|\sqrt{\alpha(t)} u(t)|_{2}^{2}+\beta(t)\|u(t)\|^{2} .
$$

Diferenciando (3.30), utilizando la ecuación (1.1) $)_{1}$, Lema 2.2 y el Teorema de la Divergencia, se obtiene

$$
\begin{aligned}
\varphi^{\prime}(t)= & 2(f(u(t)), u(t))-2\|u(t)\|_{1, p}^{p} \\
& +\left|\sqrt{\alpha^{\prime}(t)} u(t)\right|_{2}^{2}+\beta^{\prime}(t)\|u(t)\|^{2} .
\end{aligned}
$$

Por $(H 4),(3.1)$ y $E(0) \leq 0$, resulta

$$
\begin{aligned}
\varphi^{\prime}(t) & \geq 2(f(u(t)), u(t))-2\|u(t)\|_{1, p}^{p} \\
& \geq 2 \sigma \int_{\Omega} F(u(x, t)) d x-2\|u(t)\|_{1, p}^{p} \\
& \geq 2\left(\frac{\sigma-p}{p}\right)\|u(t)\|_{1, p}^{p}-2 \sigma E(0) \\
& \geq 2\left(\frac{\sigma-p}{p}\right)\|u(t)\|_{1, p}^{p}
\end{aligned}
$$

Tomando $D_{0}$ la constante óptima de la Desigualdad de Sobolev-Poincaré $|u|_{p} \leq D_{0}\|u\|_{1, p}$, $\forall u \in W_{0}^{1, p}(\Omega)$ y desde que $|v|_{2} \leq|\Omega|^{\frac{1}{2}-\frac{1}{p}}|v|_{p}, \forall v \in L^{p}(\Omega)$, se tiene

$$
\varphi^{\prime}(t) \geq\left(\frac{\sigma-p}{p}\right) D_{0}^{-p} \operatorname{mín}\left\{D_{0}^{p}, 1\right\}|\Omega|^{-\left(\frac{p-2}{2}\right)}\left[|u(t)|_{2}^{p}+\|u(t)\|^{p}\right] .
$$

Por las hipótesis $(H 1)-(H 2)$, se tiene $\alpha(x, t) \leq\|\alpha\|_{L^{\infty}\left(0 . \infty: L^{\infty}(\Omega)\right)}$ c.t.p. para $(x, t) \in \Omega \times[0, \infty[$ y $\beta(t) \leq\|\beta\|_{L^{x(0, \infty)}}$ c.t.p. para $t \in\left[0,+\infty\left[\right.\right.$. Desde que $(a+b)^{r} \leq 2^{r-1}\left(a^{r}+b^{r}\right), \forall a, b \geq 0, \forall r \geq 1$, de $(3.30)$ se obtiene

$$
\begin{aligned}
\varphi^{\frac{p}{2}}(t) & \leq C_{\alpha \beta}^{\frac{p}{2}}\left[|u(t)|_{2}^{2}+\|u(t)\|^{2}\right]^{\frac{p}{2}} \\
& \leq 2^{\frac{p-2}{2}} C_{\alpha \beta}^{\frac{p}{2}}\left[|u(t)|_{2}^{p}+\|u(t)\|^{p}\right]
\end{aligned}
$$


donde $C_{\alpha \beta}:=\operatorname{máx}\left\{\|\alpha\|_{L^{\infty}\left(0, \infty ; L^{\infty}(\Omega)\right)},\|\beta\|_{L^{\infty}(0, \infty)}\right\}$. Por (3.32), de (3.31) se obtiene

$$
\varphi^{\prime}(t) \geq K \varphi^{\frac{p}{2}}(t)
$$

donde $K:=\left(\frac{\sigma-p}{p}\right) D_{0}^{-p} \min \left\{D_{0}^{p}, 1\right\}|\Omega|^{-\left(\frac{p-2}{2}\right)} 2^{-\left(\frac{p-2}{2}\right)} C_{\alpha \beta}^{-\frac{p}{2}}$. De (3.33), resulta

$$
\varphi^{\frac{p-2}{2}}(t) \geq \frac{\varphi^{\frac{p-2}{2}}(0)}{1-\left(\frac{p-2}{2}\right) \varphi^{\frac{p-2}{2}}(0) K t}
$$

y de aquí, existe un tiempo finito $T_{*}$ tal que $\lim _{t \rightarrow T_{*}^{-}} \varphi(t)=+\infty$ y la cota superior de $T_{*}$ es estimada por (3.29). Con todo esto se finaliza la demostración del Teorema 3.7.

Observación 3.8. Si las funciones $\alpha \equiv 0$ ó $\beta \equiv 0$ pero no ambas, y se cumplan las hipótesis de los Teoremas 3.6 o 3.7, entonces se obtienen resultados similares a estos Teoremas, usando las mismas estrategias seguidas en su prueba.

Observación 3.9. Supongamos que se cumplan $\beta \equiv 0, f(u)=|u|^{\sigma-2} u, 2 \leq p<\sigma<\frac{p(n+2)}{n}$, y las hipótesis $(H 1)$ y $(H 6)$. Si $u$ es una solución del problema (1.1) sobre el intervalo maximal $\left[0, T_{\text {máx }}[\right.$ con dato inicial no cero $u_{0} \in W_{0}^{1, p}(\Omega)$, y que satisface

$$
E(0) \leq 0
$$

entonces $T_{\text {máx }}<+\infty$, es decir que $u$ no puede ser extendida sobre $[0,+\infty[$ como solución del problema (1.1). Además el tiempo finito $T_{\text {máx }}$ es estimado por

$$
T_{\text {máx }} \leq \frac{\sigma|\Omega|^{\frac{\sigma-2}{2}} C_{\alpha}^{\frac{\sigma}{2}}}{(\sigma-2)(\sigma-p)\left|\sqrt{\alpha(0)} u_{0}\right|_{2}^{\sigma-2}}
$$

donde $C_{\alpha}:=\|\alpha\|_{L^{\infty}\left(0, \infty ; L^{\infty}(\Omega)\right)}$ y $|\Omega|$ es la medida de $\Omega$. Este resultado se obtiene de manera similar que la prueba del Teorema 3.7. 


\section{REFERENCIAS BIBLIOGRÁFICAS}

[1] Ball, J. M., Remarks on blow-up and nonexistence theorems for nonlinear evolution equations, Quart. J. Math. Oxford (2), 28 (1977), 473-486.

[2] Kalantarov, V. and O. A. Ladyzhenskaya, The occurence of collapse for quasilinear equation. of parabolic and hyperbolic types, J. Sov. Math. 10 (1978), 53-70.

[3] Ladyzhenskaya, O. A., Solonnikov, V. A. and Uralceva, N. N., Linear and Quasilinear Parabolic Equations, "Nauka", Moscow, 1967; English transl., Trans. Math. Monographs, 23, American Mathematical Society, Providence, Rhode Island, 1968.

[4] Levine, H. A., Some nonexistence and instability theorems for solutions of formally parabolic equations of the form $P u_{t}=-A u+F(u)$, Arch. Rational Mech. Anal. 51 (1973), 371-386.

[5] Messaoudi S. A., A note on blow up of solutions of a quasilinear heat equation with vanishing initial energy, Math. Nachr., J. Math. Anal. Appl. 273 (2002), 243-247.

[6] Quispe Méndez, T., Solución local para una ecuación del calor degenerada no lineal, Pesquimat Revista de la F. C. M. de la Universidad Nacional Mayor de San Marcos Vol. XI - No 2, pp. 56-70, Lima - Perú. Octubre 2008.

[7] Samarskii, A.A., Galaktionov, V.A., Kurdyumov, S.P. y Mikhailov, A.P. Blow-up in problems for quasilinear parabolic equations. Nauka, Moscow, 1987 (in Russian). English transl.: Walter de Gruyter, Berlin, 1995.

[8] Tan, Z., The reaction-diffusion equation with Lewis function and critical Sobolev exponent, J. Math. Anal. Appl., 272 (2002) (2), 480-495.

[9] Tsutsumi, M., Existence and nonexistence of global solutions for nonsilinear parabolic equations, Publ. RIMS, Kyoto Univ., 8 (1972/73), 211-229.

[10] Vitillaro, E., Global nonexistence theorems for a class of evolution equations with dissipation, Arch. Rational Mech. Anal., 149 (1999), 155-182.

[11] Zhou Y., Global nonexistence for a quasilinear evolution equation with a generalized Lewis function, J. Anal. Appl. 24 (2005)(1),179-187. 